\section{Early postoperative gastric enteral nutrition improves gastric emptying after non- complicated cardiac surgery}

\author{
ALAN ŠUSTIĆ • MARKO ZELIĆ • IGOR MEDVED • JADRANKO SOKOLIĆ
}

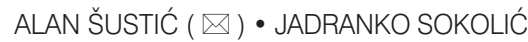
Department of Anesthesiology and ICU, University Hospital Rijeka T.Strižića 3, Rijeka 51000

Croatia, Europe

Phone/fax: + + 38551218407

e-mail: alan.sustic@medri.hr

MARKO ZELIĆ • IGOR MEDVED Department of Cardiac Surgery, University Hospital Rijeka, Rijeka, Croatia

\begin{abstract}
Background: Postoperative intragastric enteral feeding in cardiac surgery patients is frequently complicated by delayed gastric emptying.

Objective: To evaluate how early postoperative gastric enteral nutrition affects gastric emptying in coronary artery by-pass graft (CABG) surgery patients.

Methods: In this prospective, randomized study a group of 40 patients treated in the intensive care unit after CABG surgery

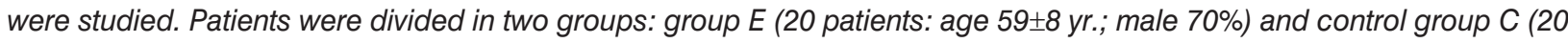
patients: age $58 \pm 10 \mathrm{yr}$; male $80 \%$ ), respectively. The paracetamol absorption test was used to evaluate gastric emptying. In group E gastric enteral nutrition begun 18 hours after surgery and 6 hours later this was stopped and paracetamol solution was administered. The patients in group $C$ received only crystalloid solutions for first 24 hours. Blood samples were obtained at $0\left(t_{0}\right), 15\left(t_{+15}\right), 30\left(t_{+30}\right), 60\left(t_{+60}\right)$ and $120\left(t_{+120}\right)$ min after administration of paracetamol.

Results: The values of plasma paracetamol concentration (PPC) at 15 and 120 min were significantly higher in group $E$ when compared with .group $C:\left(t_{+15}\right) 3.3 \pm 2.5$ vs. $1.7 \pm 1.9$ and $\left(t_{+120}\right) 5.2 \pm 2.8$ vs. $3.3 \pm 1.6(p<0.05)$. The PPC values at 30 and 60 min were higher, but not significantly, in group $E$ vs. group $C$ : $\left(t_{+30}\right) 3.7 \pm 2.0$ vs. $2.9 \pm 2.7$ and $(t+60) 5.1 \pm 3.2$ vs. $3.9 \pm 3.5$ (p $=N S)$. The area under the PPC curve was $429 \pm 309$ in the $E$ group vs. $293 \pm 204$ in the group $C(p<0.05)$.

Conclusion: Early postoperative gastric administration of nutritients after CABG surgery stimulates gastric emptying.
\end{abstract}

Key words:

Early postoperative enteral nutrition; gastric emptying

\section{Introduction}

Over the past few years, the advantages of early enteral feeding over delayed enteral feeding and over conventional total parenteral nutrition was noticed. It has been found to preserve the integrity of the gut mucosa, reduce bacterial translocation, stimulate the host defense mechanisms and improves outcome $(1,2,3)$.

Recently, it has been demonstrated that early enteral feeding in multiple injury cases diminishes gastric intol- erance towards food and promotes earlier establishment of motility in the gastroduodenal segment of the digestive tract (4). Nevertheless, we could not find any data in relevant literature concerning the impact of early postoperative enteral feeding on gastric motility in patients after cardiac surgery. The aim of this study was to gain an understanding of how early postoperative gastric enteral nutrition affects gastric emptying in patients after the coronary artery by-pass graft (CABG) surgery.

\section{Materials and methods}

The investigation was designed as a prospective, randomized, clinical study. All patients were preoperatively informed about the study protocol and they ag-reed to participate. The protocol was approved by the Ethics Committee of the University of Rijeka.

Forty patients treated in the cardiac surgery ICU, following the CABG surgery, were included in the study. Exclusion criteria for the study were: anamnestic data about diseases of gastroduodenal part of digestive tract or an endoscopic findings confirming gastric or duodenal ulceration in last five years; loss of weight of over $10 \%$ in last three months or extreme obesity (BMI > 35), diabetes mellitus, preoperative elevated bio- 
Table 1. The age, severity of illness (SAPS II), sex, body mass index (BMI), duration of cardiopulmonary by-pass and surgical procedure and overall quantity of fentanyl used during the surgical procedure $(\mathrm{mg})$ in the control group and E group (early postoperative gastric supply of nutritients).

\begin{tabular}{llll}
\hline & Control group & Group E & P value \\
\hline Age (yr.) & $58 \pm 10$ & $59 \pm 8$ & NS \\
\hline Gender (male; \%) & $14(70 \%)$ & $16(80 \%)$ & NS \\
\hline SAPS II & $21 \pm 7$ & $20 \pm 9$ & NS \\
\hline BMl & $30.4 \pm 3.5$ & $28.8 \pm 2.4$ & NS \\
\hline
\end{tabular}

Duration of

cardiopulmonary

by-pass (min)

$95 \pm 13$

$98 \pm 18$

NS

Duration of surgical

procedure (min)

$181 \pm 34$

$177 \pm 45$

NS

Overall quantity of used

fentanyl used during the

surgical procedure (mg)

$2.5 \pm 0.3$

$2.4 \pm 0.5$

NS

chemical parameters of hepatic (ASAT, ALAP, gammaGT and bilirubin) or renal function (urea, creatinine), preoperative intake of drugs which could influence gastric motility (cisapride, methoclopramide, erythromycin, dopamine in doses $>2 \mu \mathrm{g} / \mathrm{kg} / \mathrm{min}$ ) or the paracetamol absorption test (e.g. NSAID). Patients with serious concomitant valvular disease, recent myocardial infarction (< 3 weeks), preoperative ejection fraction $<35 \%$ and intraoperative use of intraaortic balloon pump due to the possible influence of hemodynamic instability on gastric motility were also excluded (5). Software-generated random numbers were assigned to the patients dividing them into the early postoperative gastric enteral nutrition (E) group (20 patients) and control (C) group (20 patients). All forty patients underwent the same preoperative procedure: intestinal enema on the day before the operation, overnight fasting and night premedication with diazepam. Surgery started in all patients after 8 hours of fasting. Anesthesia, with etomidate as an induction agent, was maintained with midazolam and fentanyl, using rocuronium for relaxation. A 3rd generation cephalosporin (ceftriaxone or cefotaxime) was used as intraoperative antimicrobial prophylaxis. All patients received a two-lumen nasogastric tube; the exact gastric position was verified radiographicaly immediately after entering ICU. All patients were monitored hemodynamically with a PA catheter (as a part of a protocol-guided perioperative care in our institution) and operated with technique of intermittent ischemia (occlusion). Postoperative mechanical ventilation lasted at least six hours using propofol for continuous sedation. The infusion of propofol was terminated after 6 hours and the patients were taken off mechanical ventilation and extubated in accordance with a senior anesthesiologist. All patients included in the study were extubated within twelve hours postoperatively and all were normothermic at the beginning of the study. Postoperative analgesia, when required, was maintained with morphine sulphate (during the study a maximum of one "bolus" of 0.01 $\mathrm{mg} / \mathrm{kg}$ i.v. was applied). The patients were divided into two groups. The age, severity of illness (SAPS II), sex, body mass index (BMI), overall quantity of used fentanyl, duration of cardiopulmonary by-pass and surgical procedure, are presented on table 1.

Enteral feeding protocol (6)

The patients in group $\mathrm{E}$ started with

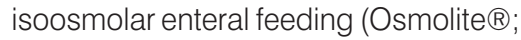
Ross) through the nasogastric tube 18 hours after CABG surgery according to the following protocol: the first 3 hours $30 \mathrm{ml} / \mathrm{h}$, next 3 hours $50 \mathrm{ml} / \mathrm{h}$, i.e. with a total of $240 \mathrm{ml}$ after 6 hours. After 6 hours of feeding (i.e. 24 hours after surgery) the gastric supply was stopped and a paracetamol solution (1000 mg/50 ml) was administrated via the nasogastric tube. Patients in group $C$ in the first 24 hours received only crystalloid solutions and after 24 hours a paracetamol solution (1000 $\mathrm{mg} / 50 \mathrm{ml}$ ) was administrated, also via the nasogastric tube.

Gastric motility (emptying) measurement

The paracetamol absorption model was used as an indirect measure of gastric motility and emptying $(7,8)$. Venous blood samples were obtained via an indwelling peripheral cannula immediately before (calibration sample) and at $15(t+15), 30(t+30), 60(t+60)$ and $120(t+120)$ min after the administration of paracetamol. Plasma concen-

Table 2.Relationship between plasma paracetamol concentration (PPC) in the control group and E group (early postoperative gastric supply of nutritients).

\begin{tabular}{lllll}
\hline & PPC15 $(\mathrm{mg} / \mathrm{L})$ & PPC30 $(\mathrm{mg} / \mathrm{L})$ & PPC60 $(\mathrm{mg} / \mathrm{L})$ & PPC120 (mg/L) \\
\hline E group & $3.26 \pm 2.5$ & $3.66 \pm 2.0$ & $5.12 \pm 3.2$ & $5.15 \pm 2.8$ \\
\hline Control group & $1.68 \pm 1.9$ & $2.94 \pm 2.7$ & $3.87 \pm 3.5$ & $3.25 \pm 1.6$ \\
\hline P value & 0.021 & $\mathrm{NS}$ & $\mathrm{NS}$ & 0.034 \\
\hline
\end{tabular}


tration of paracetamol was determined using an immunological method including fluorescence polarization (TDx $\AA$ acetaminophen, Abbott Laboratories, North Chicago, IL, USA). Paracetamol absorption was assessed from the plasma paracetamol concentration (PPC), peak paracetamol plasma levels (Cmax), and the area under the paracetamol concentration curve from 0 to 120 min (AUC120) calculated by using the trapezoidal model.

Statistical analysis

All values are presented as mean value \pm standard deviation. Statistical analysis was performed with software Statistica 6.0 (StatSoft. inc.), using Chisquare test for compared qualitative baseline variables between groups and Mann-Whitney U or one-way ANOVA tests for comparisons of quantitative variables of unpaired samples.

\section{Results}

The patients from both groups did not differ in respect to their age, sex, BMl, the duration of cardiopulmonal by-pass and duration of surgery (table 1). The results are presented in tables 2 and 3 . All values of PPC as well as the values of Cmax and AUC120 obtained during this study were higher in patients from group E. Statistically significant increase in the value of AUC 120 and PPC after 15 and 120 min. $(p<0.05)$ was noticed.

\section{Discussion}

This study focuses on one question only: does early postoperative gastric enteral nutrition improve gastric emptying in patients following cardiac surgery? From the results in this study, it is noticeable that patients undergoing the early postoperative gastric enteral nutrition protocol, after 24 hours (that is 6 hours after the beginning of enteral feeding), when $240 \mathrm{ml}$ of enteral formula and $1000 \mathrm{mg}$ of paracetamol was given, had a significantly higher concentration of paracetamol in the plasma in the next 120 min when compared with the control group. As groups were reciprocally comparable, and due to the fact that group $E$ had higher values

Table 3. Peak paracetamol plasma levels (Cmax), and the area under the paracetamol concentration curve from 0 to $120 \mathrm{~min}$ (AUC120) in the control group and E group (early postoperative gastric supply of nutritients).

\begin{tabular}{lll}
\hline & C max $(\mathrm{mg} / \mathrm{L})$ & AUC 120 \\
\hline E group & $5.15 \pm 2.8$ & $429 \pm 309$ \\
\hline Control group & $3.87 \pm 3.5$ & $293 \pm 204$ \\
\hline P value & NS & $<0.026$ \\
\hline
\end{tabular}

of PPC and AUC120, we could conclude that early postoperative gastric nutrition stimulates gastric motility and emptying.

It has been demonstrated that early enteral feeding, after multi-trauma, supports the motility of the gastroduodenal segment (4). The results of the present study show that the effect of early enteral nutrition is probably the same in patients with uncomplicated CABG surgery. Therefore, early postoperative gastric enteral nutrition might be an important factor in decreasing postoperative gastric hypo motility and gastric paresis with subsequent intolerance of enteral formula, which is not a rare observation in critically ill patients, nor in patients after cardiac surgery $(5,9)$. Postoperative intolerance of enteral food in cardiac surgery patients is caused by various factors, for example the use of certain anesthetics, opiates, vasoactive drugs and postoperative mechanical ventilation. It may also be a consequence of perioperative splanchnic hypoperfusion or of the use of extracorporal circulation (5). Although enteral feeding is the method of choice in nutritional treatment of surgical patients, due to postoperative intolerance of liquids enteral formula it is often not established adequately. This contributes to the insufficient intake of calories and greater postoperative complications, further adding to cost of treatment (10-12). In order to treat gastric hypo motility and intolerance, pro-motility drugs and small bowel tube feeding were used (13-15). However, in routine clinical use, bearing in mind economic and rational approach, and also to avoid side effects, pro-motility drugs are exclusively used in treating already existing gastric intolerance but not for the prevention of postoperative gastric hypomotility $(13,14)$. The post-pyloric placement of feeding tube requires certain clinical experience and is technically demanding and time-consuming. In comparison to the simple gastric placement, post-pyloric placement of a smallbore feeding tube is more expensive and burdened with a higher percent of complications and unsuccessful attempts. It should be stressed that a recent metaanalysis of Marik and Zaloga did not indicate any clinical benefits of post-pyloric versus gastric tube feeding in a mixed group of critically ill patients, including medical, neurosurgical, and trauma ICU patients (15). Thus, in every day clinical routine, a nasogastric tube should be utilized as a standard approach to the gastrointestinal tract with gastric feeding as the usual way of enteral nutrition. In our study we used nasogastric feeding tubes and the presented results demonstrate that early postoperative enteral feeding stimulate gastric motility and emptying in uncomplicated cardiosurgical patients.

However, the study has certain important limitations. Firstly, it excludes all cases with perioperative circulatory failure as well as seriously ill cardiac patients i.e. those with an increased risks of developing gastric paresis and gastric intolerance $(5,9)$. In our opinion, before determining definitive postoperative enteral feeding guidelines, a similar study should be done on cardiosurgical patients with significant hemodynamic instability and/ or with verified (subsequent) splanchnic hypoperfusion. Secondly, a relatively small number of patients was included in the study and the paracetomol test is a somewhat robust method for evaluating gastric emptying. In our opinion this is 
the main reason for such insignificant difference in the values of PPC after 30 and 60 minutes, as well as in the values of Cmax.
In conclusion we have shown that early postoperative gastric supply with liquid isoosmolar enteral formula probably improves gastric emptying after uncomplicated cardiac surgery and may present a mode to prevent postoperative gastric hypomotility with subsequent gastric intolerance.

Research support:

Ministry of Science of the Republic of Croatia (Project: 0062078)

\section{REFERENCES}

1. P. Jolliet, C. Pichard, G. Biolo, R. Chiolero R, G. Grimble, X. Levarve, et al: "Enteral nutrition in intensive care patients: a practical approach. Working Group on Nutrition and Metabolism, ESICM. European Society of Intensive Care Medicine", Intensive Care Med, Vol. 24, (1998), pp. 848-859.

2. M. Braga, L. Gianotti, A. Vignali, A. Cestari, P. Bisagni, V DiCarlo: "Artificial nutrition after major abdominal surgery: impact of route of administration and composition of the diet", Crit Care Med, Vol. 26, (1998), pp. 24-30.

3. G. Minard, K.A. Kudsk: "Is early feeding beneficial? How early is early?", New Horiz, Vol. 2, (1994), pp. 156-163.

4. L. Kompan, B. Kremžar, E. Gadžijev, M. Prosek: "Effects of early enteral nutrition on intestinal permeability and the development of multiple organ failure after multiple injury", Intensive Care Med, Vol. 25, (1999), pp. 157-161.

5. M.M. Berger, M. Berger-Gryllaki, P.H. Weisel, J.P. Revelly, M. Hurni, C. Cayeux, et al: "Intestinal absorption in patients after cardiac surgery", Crit Care Med, Vol. 28, (2000), pp. 2217-2223

6. A. Šustić, Ž. Krznarić, M. Uravić, Ž. Fučkar, D. Štimac, Ž. Župan: "Influence on gallbladder volume of early postoperative gastric supply of nutrients", Clin Nutr, Vol. 19, (2000), pp. 413-416.

7. M. Sanaka, Y. Kuyama, M. Yamanaka: "Guide for judicious use of the paracetamol absorption technique in the study of gastric emptying rate of liquids", J Gastroenterol, Vol. 33, (1998), pp. 785-791.

8. M.M. Tarling, C.C. Toner, P.S. Withington, M.K. Baxter, R. Whelpton, D.R. Goldhill: "A model of gastric emptying using paracetamol absorption in intensive care patients", Intensive Care Med, Vol. 23, (1997), pp. 256-260.

9. P.E. Marik, G.P. Zaloga: "Early enteral nutrition in acutely ill patients: a systematic review", Crit Care Med, Vol. 29, (2001), pp. $2264-2270$.

10. S. Adam, S. Batson: "A study of problems associated with the delivery of enteral feed in critically ill patients in five ICUs in UK", Intensive Care Med, Vol. 23, (1997), pp. 261-266.

11. M. Braga, L. Gianotti, O. Gentilini, V. Parisi, C. Salis, V. DiCarlo: "Early postoperative enteral nutrition improves gut oxygenation and reduces costs compared with total parenteral nutrition", Crit Care Med, Vol. 29, (2001), pp. 242-248.

12. B. De Jonghe, C. Appere-De-Vechi, M. Fournier, B. Tran, J. Merrer, J.C. Melchior, et al: "A prospective survey of nutritional support practices in intensive care units patients: what is prescribed? What is delivered?", Crit Care Med, Vol. 29, (2001), pp. 8-12.

13. R. MacLaren: "Intolerance to intragastric enteral nutrition in critically ill patients: complications and management", Pharmacotherapy, Vol. 20, (2000), pp. 1486-1498.

14. R. MacLaren, D.A. Kuhl, J.M. Gervasio, R.O. Brown, R.N. Dickerson, T.N. Livingston, et al: "Sequential single doses of cisapride, erytromycin, and metoclopramide in critically ill patients intolerant to enteral nutrition: a randomized, placebo-controlled, crossover study", Crit Care Med, Vol. 28, (2000), pp. 438-444.

15. P.E. Marik, G.P. Zaloga: "Gastric versus post-pyloric feeding: a systematic review", Critical Care, Vol. 7, (2003), pp. R46-R51. 